\section{When makes you unique: Temporality of the human brain fingerprint}

\author{
Dimitri Van De Ville $e^{1,2,3}$, Younes Farouj ${ }^{1,2}$, Maria Giulia Preti ${ }^{1,2,3}$, \\ Raphaël Liégeois $^{1,2}$, Enrico Amico ${ }^{1,2 *}$
}

\begin{abstract}
The extraction of "fingerprints" from human brain connectivity data has become a new frontier in neuroscience. However, the time scales of human brain identifiability are still largely unexplored. We here investigate the dynamics of brain fingerprints along two complementary axes: (i) What is the optimal time scale at which brain fingerprints integrate information and (ii) when best identification happens. Using dynamic identifiability, we show that the best identification emerges at longer time scales; however, short transient "bursts of identifiability," associated with neuronal activity, persist even when looking at shorter functional interactions. Furthermore, we report evidence that different parts of connectome fingerprints relate to different time scales, i.e., more visualsomatomotor at short temporal windows and more frontoparietal-DMN driven at increasing temporal windows. Last, different cognitive functions appear to be meta-analytically implicated in dynamic fingerprints across time scales. We hope that this investigation will advance our understanding of what makes our brains unique.
\end{abstract}

\section{INTRODUCTION}

In the 17th century, physician Marcello Malpighi observed the existence of distinctive patterns of ridges and sweat glands on fingertips (1). This key observation led to a long and continuing quest for ways to uniquely identify individuals based on fingerprints, a technique massively used until today. In the modern era, the concept of fingerprinting has expanded into other biometric data, such as voice recordings and retinal scans, among many others (2). It is only in the past few years that technologies and methodologies have achieved high-quality measures of an individual's brain to the extent that personality traits and behavior can now be characterized. The most insightful correlates emerged from the investigation of structural and functional connectivity (FC) that can be modeled and analyzed using network science, an area of research usually referred to as brain connectomics (3). For the structural connectome, white matter pathways between pairs of brain regions are quantified using diffusionweighted imaging and referred to as structural connectivity (SC). For the functional connectome, temporal statistical dependencies between activity time courses of these regions are taken from functional magnetic resonance imaging (fMRI) to define FC $(3,4)$. The most common paradigm for FC is resting-state fMRI during which subjects in the scanner are not engaging in a particular task (5).

The concept of "fingerprints of the brain" is very novel $(6,7)$ and has been boosted because of the seminal work by Finn et al. (6) in 2015. They were among the first to show that, to a great extent, it is possible to robustly identify a "target" subject's functional connectome from a database of FCs, simply by computing the connection-wise (Pearson) correlation between the target FC and those in the database. The success rate (SR) of this identification procedure was above $90 \%$ for resting-state sessions and ranged between 54 and $87 \%$ when considering task-task and task-rest sessions (6). Therefore, an individual's functional brain connectivity profile is both unique and reliable, similar to a fingerprint, and it is possible, with near-perfect

\footnotetext{
'Institute of Bioengineering, Center for Neuroprosthetics, EPFL, Geneva, Switzerland ${ }^{2}$ Department of Radiology and Medical Informatics, University of Geneva (UNIGE), Geneva, Switzerland. ${ }^{3}$ CIBM Center for Biomedical Imaging, Vaud, Switzerland. *Corresponding author. Email: enrico.amico@epfl.ch
}

accuracy in many cases, to identify an individual among a large group of subjects solely on the basis of her or his connectivity profile. These findings incentivized human neuroimaging studies to move from inferences at the population level to results that apply to the single-subject level, i.e., by acknowledging and exploiting properties of functional network organization unique to an individual across cognitive tasks and resting state $(8,9)$, by relating such functional organization to behavioral phenotypes in both health and disease (10), or even by implementing ways for maximizing and denoising fingerprints from brain data (11).

Recently, several studies have started to look into the temporal aspect of brain fingerprints. Such ventures into the "temporal domain" of brain fingerprints are important for two main reasons. First, it might provide insights into whether the connectivity patterns needed for brain identification are also the ones mostly linked to the time scale of the different cognitive processes taking place in a human brain. Studies have explored the dynamical organization of brain activity across temporal scales $(12,13)$ and have shown that there might be a hierarchy of progressively longer temporal receptive windows in the human brain, from sensory to higher-order cognitive processes (14). Hence, investigating how brain fingerprints relate to time scales of cognition might advance our knowledge on the individuality of brain functions, which can have important implications in the emerging fields of precision medicine and brain connectomics of disease (10).

Second, studying temporality of brain fingerprints may improve our understanding of whether subject identification based on functional brain data is a continuum or possibly preferential for specific windows in time, where one's brain is more identifiable. To address these aims, researchers have started investigating the effect of scan length on brain fingerprinting $(11,15)$, how brain fingerprints vary across weeks and months (16), and finally using dynamic FC to find associations between fluctuations in brain's functional systems and cognitive performance $(17,18)$. In particular, a first exploration by Liu et al. (17), using dynamic FC analysis, showed that individual variability was mainly spread across three higher-order cognitive systems (i.e., default mode, dorsal attention, and frontoparietal) and two primary systems (i.e., visual and sensorimotor). Notably, the spatial patterns of these dynamic characteristics of brain connectivity could 
successfully identify individuals with high accuracy and even predict individual higher cognitive performance (e.g., fluid intelligence and executive function) (17). Together, these first findings confirmed the great potential of tapping into the inherent functional dynamics of individual brain networks.

Yet, these first explorations raised new questions. In particular, what exactly is the information encoded in brain connectomes that ultimately leads to correctly differentiating someone's connectome from anybody else's? In other words, what makes our brains unique? More specifically, related to the temporality of FC-based fingerprinting, is the brain more unique at some moments, and what is the temporal extent needed for a fingerprint to unfold?

Here, we address these questions by tapping into the temporal dynamics of human brain connectivity. We use dynamic FC techniques to explore the time scales of brain identification, i.e., when and over which duration do these unique fingerprints originate, and which brain areas are most responsible for this. We demonstrate that optimal fingerprints manifest at a time scale of $200 \mathrm{~s}$ based on dynamic functional connectomes (dFCs). Nonetheless, unique individual "snapshots" of brain connectivity emerge at much shorter time scales. In addition, snapshots at different time scales reveal specific connectivity patterns in terms of regions and functional networks, which show how fMRI blood oxygenation level dependent (BOLD) fluctuations relate to different types of underlying neuronal events. Moreover, when looking at different areas in the brain fingerprints, we noticed that subcortical regions are the fastest ones for individual identification; visual and somatomotor regions appear right after; ultimately, at slower time scales, frontoparietal and default mode network (DMN) emerge. Last, a meta-analytical investigation revealed that brain fingerprints can be associated with behaviorally relevant arrangement, revealing a complex gradient of relationship between the time scale of fingerprinting and transitions from sensory behavioral traits to higher-order cognitive functions. In summary, dynamic FC methods allowed us to investigate the temporality of brain fingerprints. We provide evidence that what makes our brains unique is multifaceted, based on when and how long. That is, individual identification is a temporally integrating and fluctuating feature of brain fingerprints.

\section{RESULTS}

We introduce "dynamic brain fingerprints" to investigate the temporality of brain fingerprinting. The general scheme for dynamic identification can be divided in three steps (Fig. 1): (i) The time scale is set by the choice of the temporal window length; (ii) sliding window $\mathrm{dFC}$ frames are computed for each window position; and (iii) the similarity of frames within-subjects and between-subjects is evaluated, with the aim to extract the best "identifiable" connectome frames, for each subject (Fig. 1; see also fig. S1). In a nutshell, the temporal exploration of human dynamic fingerprints can be decomposed according to two complementary axes: the time scale of brain identification or how long it takes for the information to optimally integrate, and the best matching time of identification or when best information is available (Fig. 1). This concept can be formally encoded into a "dynamic identifiability matrix" (fig. S1), in which the blocks represent within-subject dFC similarity and off block-diagonal elements contain the information on the between-subject dFC similarity (fig. S1; see also Materials and Methods for details).

We explored these temporality aspects of human fingerprints across different time scales (window lengths) on the 100 unrelated subjects of the Human Connectome Project (HCP) dataset. We started by selecting six different window lengths $(7.2,36,72,144,288$, and $576 \mathrm{~s}$, with a fixed sliding window step of $7.2 \mathrm{~s}$ ) and explored dynamic differential identifiability (dIdiff) and SR (6) across the different time scales (Fig. 2; see also Materials and Methods for details on the implementation). In a nutshell, $d$ Idiff is an extension of the differential identifiability score (11), which originally evaluated the difference between each subject's FC self-similarity against the other subjects' FCs. In this work, because of dFC evaluation, we extended this concept to the test/retest matching within and between the set of dynamic "frames" of connectivity estimated. Furthermore, we used SR as a complementary score to $d$ Idiff to assess the likelihood to correctly identify individuals from their dynamic FC frames.

The dynamic differential identification increases steadily with longer window lengths, as well as the corresponding SRs (Fig. 2). This is expected because we rely upon more time points for dFC computation, increasing the stability of the FC profiles across testretest sessions. However, as $d$ Idiff and SR scores reach values comparable to the ones obtained with static connectivity analysis $(6,11)$, clearly noticeable diagonal blocks respecting the subject boundaries start appearing before the maximal $d$ Idiff window length (Fig. 2). This suggests that, even at shorter time scales, there exist specific dynamic fingerprints able to reliably link test-retest sessions.

We then explored whether there were specific individual dFC frames that would be driving the dynamic identification. We therefore ranked the frames based on how good they could represent the subject (or $d$ Iself; see Materials and Methods for details) across test-retest and evaluated how good these dFC frames could also separate between subjects, via $d$ Iothers. Figure 3 shows that few FC frames can drive identifiability, especially at shorter window lengths (steeper curves; Fig. 3A). Note that, although the $d$ Iself behavior is expected because we based our $\mathrm{dFC}$ frames ranking on it, the fact that $d$ Iothers (and consequently $d$ Idiff) might follow the same trend is not trivial (Fig. 3A). In other words, there exist pairs of frames where an individual is maximally similar with itself ( $d$ Iself panel; Fig. $3 \mathrm{~A})$, which leads to a larger dIdiff than for the static case.

When looking at the variability of the top frame for identification across subjects, while median values keep stable across time scales and mostly around within-network connectivity (Fig. 3C), one can notice the emergence of characteristic spatial patterns of connectivity becoming more homogeneous in the population (Fig. 3B). The variability in dynamic connectivity starts most prominent between sensory (i.e., visual and somatomotor) and higher-order (i.e., dorsal attentional, frontoparietal, and DMN) networks at shorter temporal windows, transitioning then toward within-network connectivity at slower time scales, mainly involving frontoparietal and DMN connectivity (Fig. 3B).

To evaluate the contribution of BOLD fluctuations that are most likely driven by neuronal activity-inducing signals, we repeated the same analysis using transient activity time courses [as in (19)] obtained as the derivative of the deconvolved BOLD signals (fig. S2). This simultaneously removes the effect of the hemodynamic response function and detects transition moments in neural activity. The same finding as before is confirmed across $d$ Iself, $d$ Iothers, and $d$ Idiff, with small fluctuations across temporal scales (fig. S2).

Given the observed spatial variation as a function of time scale, we investigated in more detail the spatial profiles of the fingerprints. Specifically, we used edgewise intraclass correlation (ICC; see Materials and Methods) to explore the FC connections most contributing to 


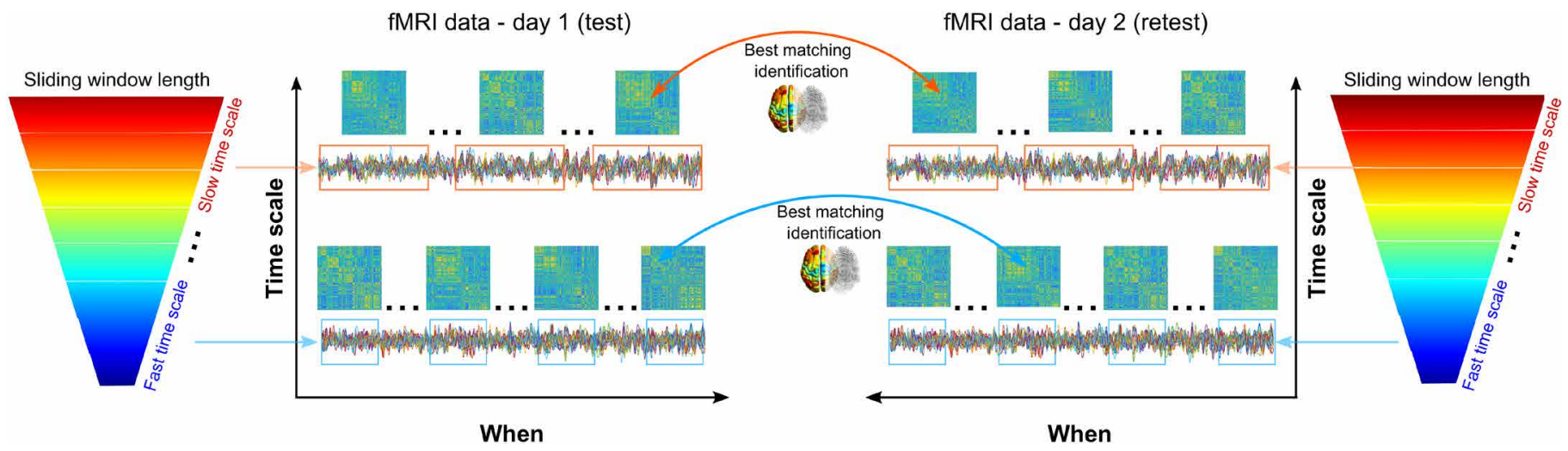

Fig. 1. Exploring dynamic brain fingerprints. Schematic of dynamic connectome identification for one subject. First, the time scale (window length) of the exploration, here depicted as a gradient cone, is set; second, dynamic FC frames are computed at each window for both test and retest fMRI data; finally, the best matching frames across test and retest data are retrieved for identification.

\section{Dynamic identification across temporal scales}

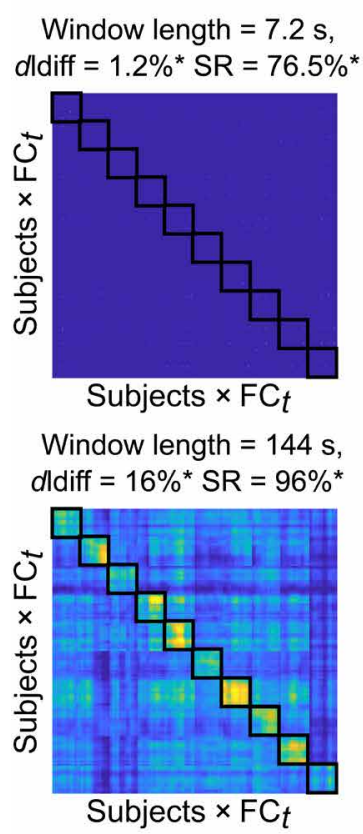

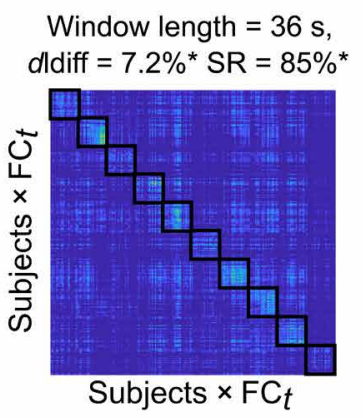

Window length $=288 \mathrm{~s}$, dldiff $=20.3 \%$ * SR $=96.5 \%$ *

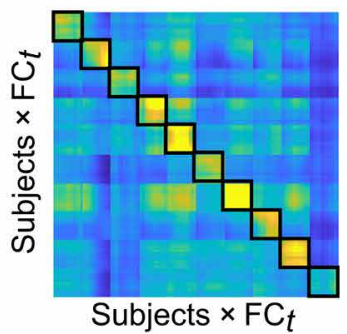

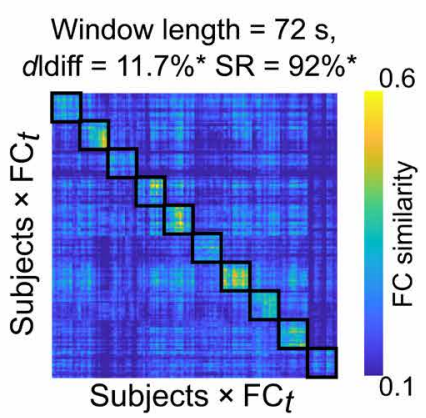

Window length $=576 \mathrm{~s}$, dldiff $=24.2 \%{ }^{*} \mathrm{SR}=96.5 \%{ }^{*} 0.6$

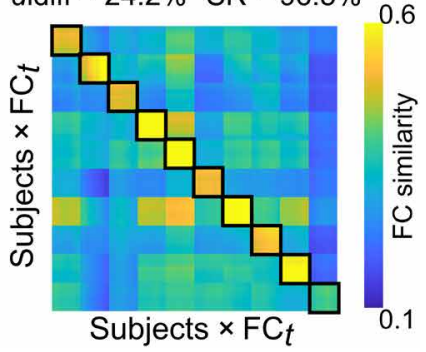

Fig. 2. Dynamic identification across temporal scales. Dynamic identifiability matrix evaluation at six different window lengths: $(7.2,36,72,144,288$, and $576 \mathrm{~s})$. To ease its visualization, the dynamic identifiability matrix in the figure was reduced to 10 sample subjects. The dynamic differential identifiability (dldiff) values and success rates (SRs) on top of each matrix provide two complementary scores of the fingerprint level of the dataset across temporal scales; the asterisks denote significance from the chance level (set at $P<0.05$, Bonferroni-corrected across the six temporal scales) from the null distributions obtained by randomizing the dynamic identifiability matrices at each time scale (see Materials and Methods for details). Black squares indicate the self-similarity of each subject's dFC frames.

brain fingerprinting across time scales. Figure 4 shows that, at fast time scales, the most reliable (i.e., with ICC >0.4) FC edges are the ones related to the connectivity between somatomotor and visual regions (Fig. 4A). As the time scale increases, "higher-order" regions start to appear, such as DMN and frontoparietal regions (Fig. 4A). Notably, no ICC patterns above 0.4 could be obtained from 100 instances of surrogate data obtained by randomly shuffling subject labels in the dataset (fig. S3; see also Materials and Methods for details on the implementation).

These results reveal a specific time scale for fingerprinting of different functional networks. When refining the temporal exploration (see Materials and Methods for details) and looking at the nodal counterpart of the ICC profiles across functional networks, one can notice that the temporal fingerprint of each functional subsystem peaks at specific times (Fig. 4B): shorter for subcortical and somatomotor connections and longer for DMN/frontoparietal ones (Fig. 4, B and C).

Last, we looked into the temporality of brain fingerprints and the link with behavior. We applied a Neurosynth meta-analysis based on 50 topic terms onto the brain fingerprint extracted at a specific temporal window, similarly to previous work $(20,21)$. We found that brain fingerprints at fast scales are associated with low-order multisensory processing, visual perception, motor/eye movements, 
A

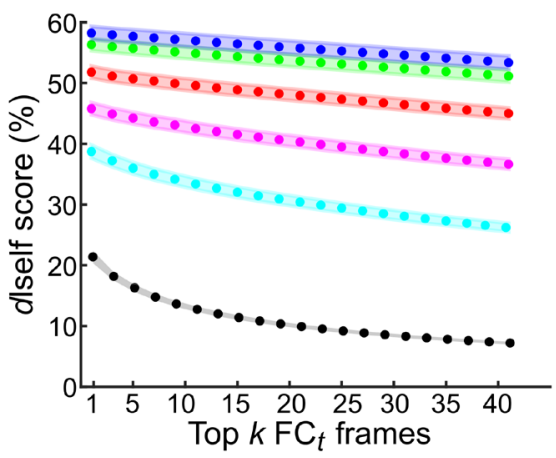

B
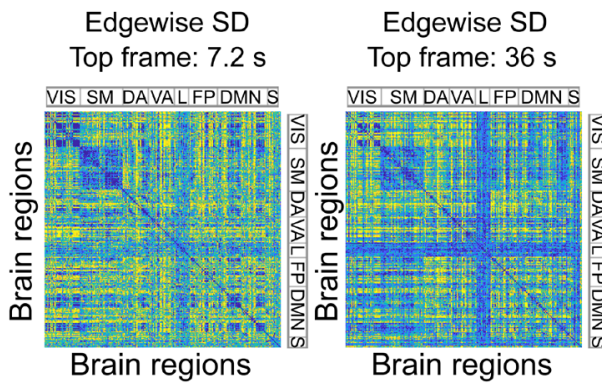$$
5
$$$$
\text { Percentile } 95
$$

C

\section{Edgewise median}

Top frame: $7.2 \mathrm{~s}$

\section{VIS SM DAVALFPDMN S}
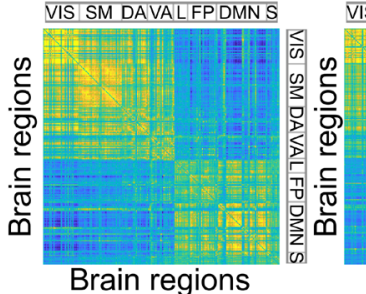

Edgewise median Top frame: $36 \mathrm{~s}$ VIS SM DAVALFPDMNS

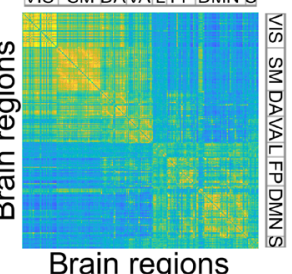

Brain regions

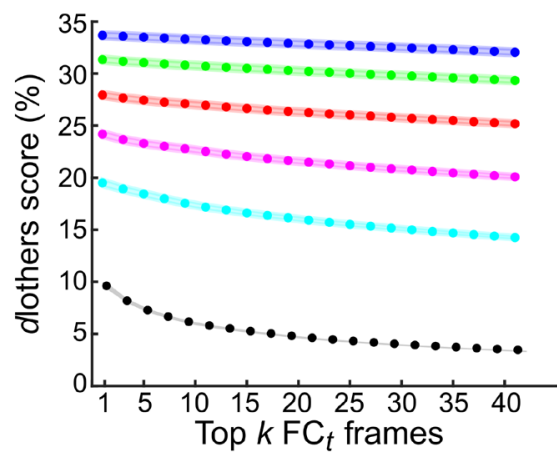

Edgewise SD

Top frame: $72 \mathrm{~s}$

VIS SM DAVALFPDMNS

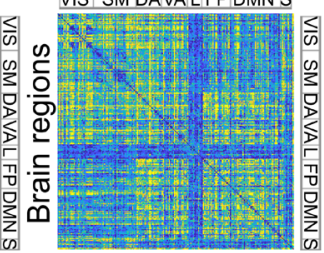

Brain regions

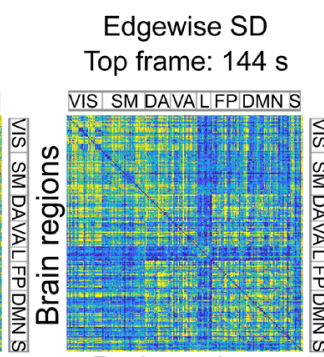

Brain regions

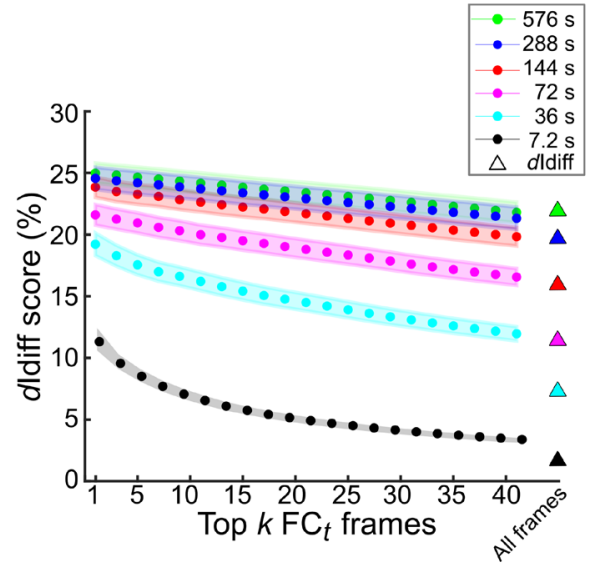

Edgewise SD

Top frame: $288 \mathrm{~s}$

VIS SM DAVALFPDMNS

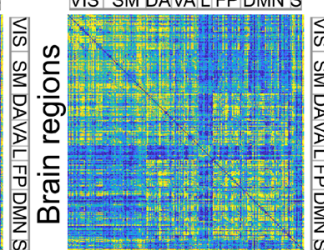

Brain regions
Edgewise SD

VIS SM DAVALFPDMNS

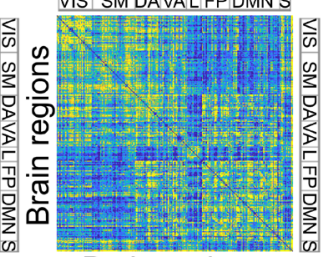

Brain regions
Top frame: $576 \mathrm{~s}$

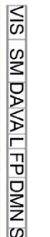

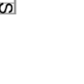


A

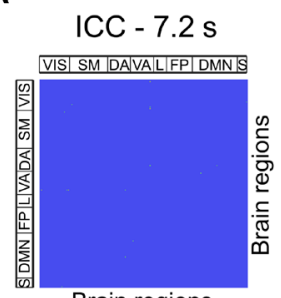

Brain regions

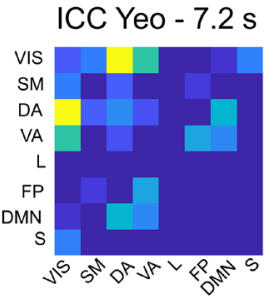

B

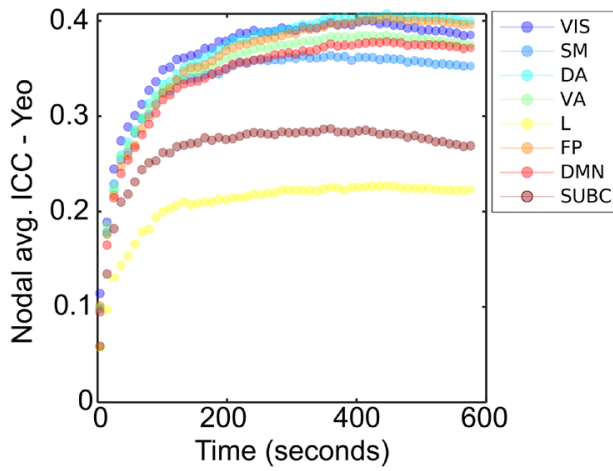

ICC - $36 \mathrm{~s}$

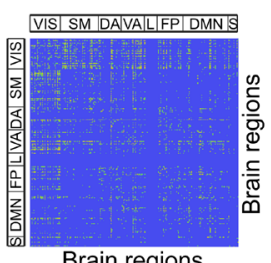

Brain regions

ICC Yeo - $36 \mathrm{~s}$

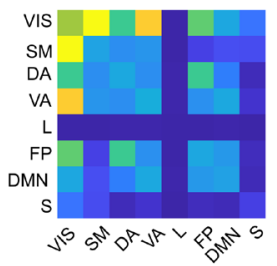

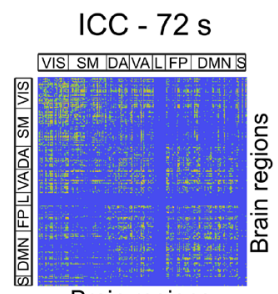

Brain regions

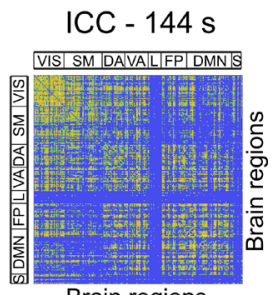

Brain regions
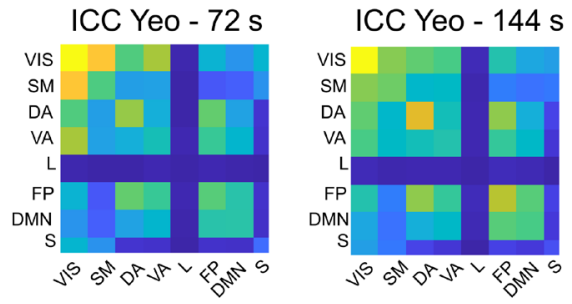

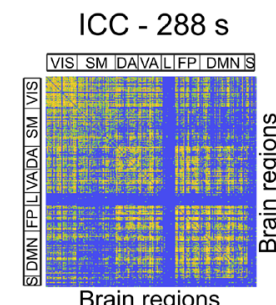

Brain regions

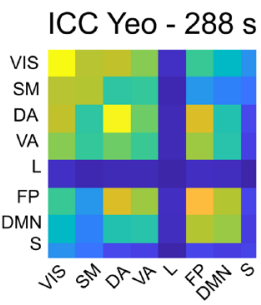

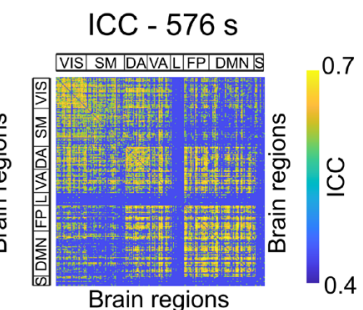

Brain regions

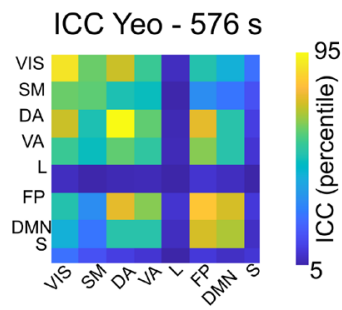

C

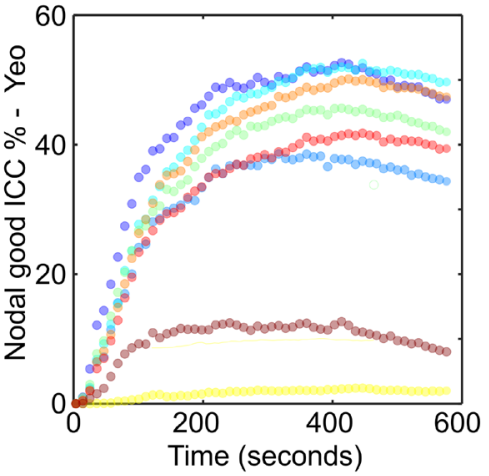

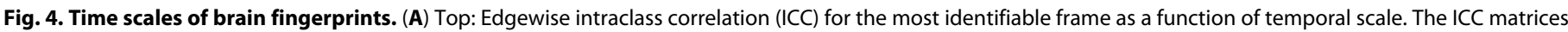

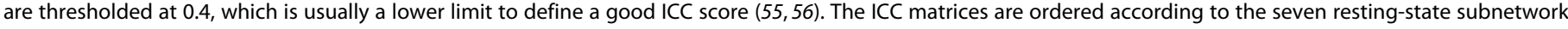

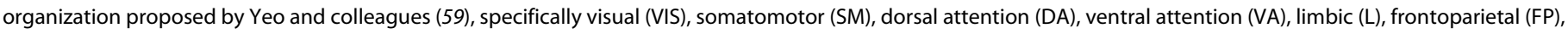

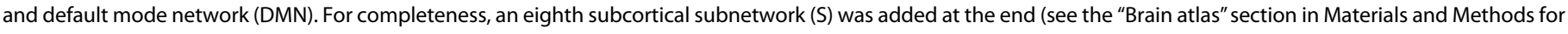

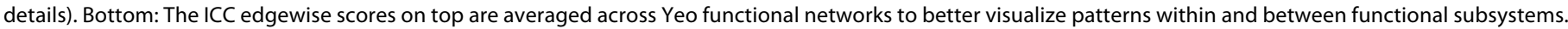

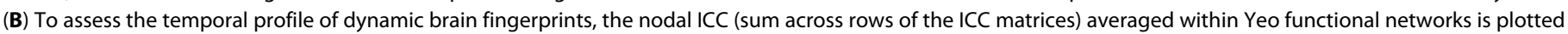

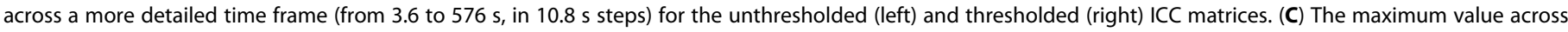

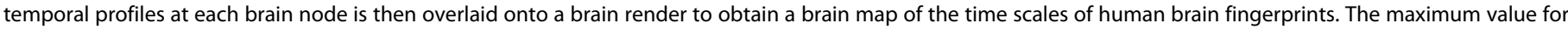

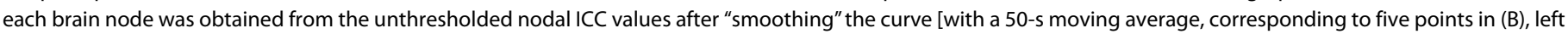
plot] to reduce sampling variability and minimize the effects of local maxima.

before. It relates to the key question: When and at which time scale functional connectomes become unique and thus identifiable?

To figure out what makes a human brain identifiable at the level of functional neuroimaging correlates, we explored the temporality of brain fingerprints along two complementary directions: (i) What is the optimal time scale at which fingerprints integrate sufficient information and (ii) when does better identification of a fluctuating pattern happen. Using the concept of dynamic identifiability (Fig. 1), we showed that the best identification emerges at longer time scales (Fig. 2), which is in line with previous work investigating the effect of scan length on individual variability $(11,15)$. However, short transient bursts of "identifiability" persist even when looking at shorter functional interactions, similarly to previous studies $(26,27)$. Even at faster time scales, very few frames suffice to identify an individual from the others (Fig. 3). However, while looking at "when" the most identifiable frames happen along the fMRI scans, we found that no significant trend emerged outside of the "boundary artifact" (i.e., half of the window size) toward the middle of the scan at longer windowlengths (fig. S4). In other words, the most identifiable frames do not seem to have a preferable time location along the resting-state fMRI scans.

These bursts of identifiability might be strongly associated with neuronal activity, as the regularized deconvolution with the hemodynamic response function did not tamper the identification rates obtained (fig. S1). It is known that mammalian cortical neurons interact in functionally relevant oscillating networks, which span across a broad frequency range (28). There is also recent evidence that episodic local field potential oscillations elicit whole-brain fMRI activity: For instance, hippocampal population burst appears temporally bounded by massive activations of association and primary cortical areas in monkeys (29). On the basis of our findings on human dynamic fingerprints, we conjecture that this burst of neuronal activity might be one of the sources of this subject specificity and therefore 

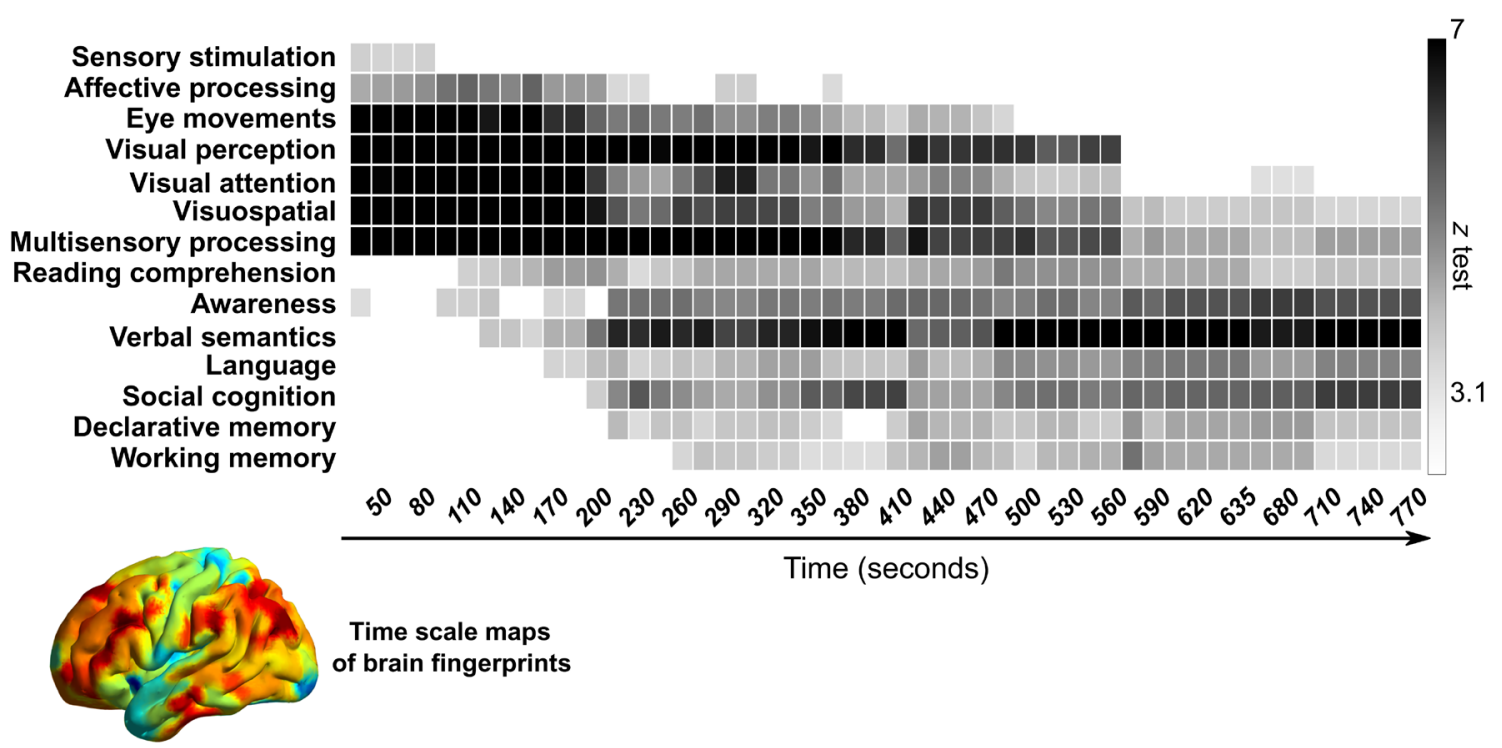

Time (seconds)

Time scale maps

of brain fingerprints

Fig. 5. Brain fingerprints are associated with behavior across time scales. The Neurosynth meta-analysis of the brain fingerprints maps across time scales (from 50 to $770 \mathrm{~s}$, in steps of $15 \mathrm{~s}$ ) shows a spectrum of association with low-sensory regions at fast time scales, ending up into higher-order processing. The brain fingerprint maps were masked by selecting only the top $25 \%$ brain nodes at each time scale.

closely related to the transient burst in identifiability observed (Fig. 3 and fig. S1).

Furthermore, previous studies $(6,11)$ showed that the main drivers of the uniqueness of each individual functional connectome reside in the brain areas devoted to higher-order cognitive functions, such as frontoparietal and DMNs. We found compelling evidence that different parts of connectome fingerprints are optimal at different time scales (Fig. 4). Each region contributes differently to fingerprinting at a specific time scale, i.e., more visual-somatomotor at short temporal windows and more frontoparietal-DMN driven with increasing temporal windows. These findings open up interesting speculation on the link between individual connectivity profiles and the information content associated with the windowed BOLD time series. Maybe higher-order cortical fluctuations contain more slow long-range information that is lost when looking at short windows within them?

Along this line of thinking, our work also provides two new insights into the relationship between resting-state connectome stability (30) and fingerprinting, which is still largely unknown. Notably, having stable connections across time and subjects seems not to be necessarily related to high brain fingerprinting. A good example is provided by the limbic network, which appears to be highly stable across subjects and scans (Fig. 3B) but poorly identifiable (Fig. 4). The limbic network is known to be highly susceptible to scanner noise/artifact due to the proximity to the sinuses and is also already known to contribute less to individual identification than other cortical networks (11). However, the limbic system is also known to be responsible for emotional processing and the processing of dangerous elements in the environment (31). The thought that a stable primitive network of emotional processing, common to all human brains, did not develop to differentiate individuals but merely to generate common (groupwise) responses to external danger is intriguing (32).

Human brain regions appear to be broadly differentiated into different aspects of behavior and cognition, and the temporal dynamics of neuronal populations across the cortex are thought to be reflective of this specialization (33-36). For example, primary sensory neurons are tightly coupled to changes in the environment, firing rapidly to the onset and removal of a stimulus and showing characteristically short intrinsic time scales. In contrast, neurons in cortical association (or transmodal) regions exhibit longer firing patterns when a person is engaged in higher-order cognitive tasks (33). Here, we hypothesized that the difference in the brain fingerprint spatial patterns might be tightly linked to the neuronal time scale of the different cognitive processes taking place in a resting human brain (Fig. 4).

The temporal scales of fingerprinting can be related to behavior in a meaningful way (Fig. 5). Using the Neurosynth (37) meta-analytic approach, we showed that there is a broad spectrum of associations with behavior. At faster time scales, human brain fingerprints are linked to multisensory stimulation, eye movements, affective processing, and visuospatial attention. At slower time scales instead, we find higher-cognitive functions, such as language and verbal semantics, awareness, declarative and working memory, and social cognition (Fig. 5). This is in line with previous work showing that early visual areas (e.g., primary visual cortex and motion-sensitive areas) exhibited high response in short temporal receptive windows, as opposed to several higher brain areas (e.g., superior temporal sulcus, precuneus, and temporal parietal junction), which was affected by information accumulated over longer time scales (14). It also corroborates recent evidence of intrinsic neuronal time scales observed in magnetoencephalography (MEG) data where core-periphery organization reflects slower-faster time scales, respectively (38). Our findings also suggest that different subtypes of spontaneous ("resting-state") cognitive processes are detectable in specific patterns of time-varying FC, depending on the chosen window. Studies of attentional fluctuations, memory reactivation, and the relationship between baseline brain activity and variation in perception have each shown that spontaneous and transient changes in BOLD FC are relevant to behavior and experience (39). By building on the extant literature, our finding reveals the link between the individual behavioral relevance 
of specific functional networks and the associated time scale at which they are manifested.

Notably, these findings are also in line with recent evidence that neuronal time scales follow cytoarchitectonic gradients across the human cortex and are relevant for cognition in both short and long terms (33). Particularly, neuronal time scales increase along the principal sensorimotor-to-association axis across the cortex and align with macroscopic gradients of gray matter myelination (T1w/T2 $\mathrm{w}$ ratio) and synaptic receptor and ion channel gene expression (33). Previous work also suggests that functional cortical networks are organized as two large ring-shaped networks (differentiated by their preferred information processing mode) (40). The first ring comprises visual, auditory, somatosensory, and motor cortices that process real-time world interactions; the second ring includes parietal, temporal, and frontal regions, with networks dedicated to cognitive functions, emotions, biological needs, and internally driven rhythms. There is evidence that the patterns of gene expression organize the cortex into two sets of regions that match the two rings (40). Overall, the correspondence between the temporal maps of dynamic brain fingerprints and the genetic/cytoarchitectonic profiles, as well as behaviorally relevant gradients (21), opens up more fascinating questions on the relationships between these gradients and human brain identifiability.

This work comes with some limitations. First, the impact of the choice of the brain atlas should be further verified. Second, we examined temporality of brain fingerprints here using sliding window analysis. Future studies should also consider other approaches, such as edgewise connectivity (41) or more advanced dynamic FC models (42). Third, as is well known, connectivity measures are highly susceptible to artifacts arising from head motion and respiratory fluctuations $(43,44)$, and these effects are even more pronounced in dynamic FC, where there are fewer time points available for a robust correlation. However, the data points with high motion present in the data did not seem to be a contributor to the differences in fingerprinting across time scales reported here (fig. S5). Notably, the main findings reported in Fig. 3 (B and C) across temporal scales could be replicated when excluding motion-affected fMRI volumes (average correlation between the SD patterns with and without scrubbing: $0.99 \pm 0.01$; median patterns: $0.988 \pm 0.001$ ) from the most identifiable dynamic FC frames. Nonetheless, future work should dig deeper on the effect of motion at shorter time scales, where these artifacts can particularly dominate.

The investigation on the relationship between transient activation and brain identifiability (fig. S2) suggests that identification is unlikely to be explained only as a by-product of hemodynamics. Hence, it would be also interesting to compare the findings from this study, which are based on fMRI resting-state data, with data coming from fMRI task analysis, or even other neuroimaging modalities, such as EEG (electroencephalography) or MEG. This would allow us to extend the range of accessible time scales across modalities for dynamic identification. This work also opens the avenue of relating functional brain fingerprints with the underlying structural architecture. Recent studies have shown that building FC matrices from (very) long resting-state fMRI sessions leads to very good proxies for SC (45). Similarly, it has been shown that longer fMRI sessions [up to a plateau (11)] improve identification. Furthermore, function-structure dependency was recently shown to follow a brain pattern extremely consistent with the gradient found here for brain fingerprints (20). Future work on identifiability and its association with SC seems therefore worth exploring.
In summary, we have here explored the temporality of the human brain fingerprint. We have shown that fingerprints are intertwined with the time scales of functional brain connectivity and possibly associated with transient bursts in brain activity. This investigation is promising based on these first findings and represents a step toward a better understanding of what and when makes our brains unique.

\section{MATERIALS AND METHODS HCP data: Functional preprocessing}

The fMRI dataset used in this work is from the HCP (www. humanconnectome.org), Release Q3. We assessed the 100 unrelated subjects ( 54 females and 46 males, mean age $=29.1 \pm 3.7$ years) as provided from the HCP 900 subjects data release $(22,46)$. Per HCP protocol, all subjects gave written informed consent to the HCP consortium. The fMRI resting-state runs (HCP filenames: rfMRI_ REST1 and rfMRI_REST2) were acquired in separate sessions on two different days, with two different acquisitions (left to right or LR and right to left or RL) per day $(47,48)$. For all sessions, data from both the LR and RL phase-encoding runs were used to calculate connectivity matrices to have four functional connectomes (one LR test-retest pair, one RL) per subject. For this study, we used the minimally preprocessed HCP resting-state data (47), with the following preprocessing steps. First, we applied a standard general linear model regression that included detrending and removal of quadratic trends; removal of motion regressors and their first derivatives; removal of white matter, cerebrospinal fluid signals, and their first derivatives; and global signal regression (and its derivative). Second, we bandpass-filtered the time series in the range of 0.01 to $0.15 \mathrm{~Hz}$. Last, the voxel-wise fMRI time series were averaged into their corresponding brain nodes of the atlas (see the next section) and then $z$-scored.

\section{Brain atlas}

We used a cortical parcellation into 400 brain regions as recently proposed by Schaefer and collaborators (49) (freely available at https://github.com/ThomasYeoLab/CBIG/tree/master/stable projects/brain_parcellation/Schaefer2018_LocalGlobal). For completeness, 16 subcortical regions and 3 cerebellar regions were also added, as provided by the HCP release (filename "Atlas_ROI2.nii.gz"), resulting in a final brain atlas of 419 brain nodes.

\section{Dynamic FC estimation}

To assess brain fingerprints across time scales, we performed sliding window analysis (42). The sliding window scheme is the following: First, a temporal window, parameterized by its length $w$, is chosen, and within the temporal interval that it spans (i.e., from time $t=1$ to time $t=w$ ), connectivity is computed between each pair of time courses as Pearson correlation coefficient, producing one instance of the "dFC" (Fig. 1A). Then, the window is shifted by a step $T$, and the same calculations are repeated over the time inter$\operatorname{val}(t=1+T, t=w+T)$. This process is iterated until the window spans the end part of the time course to obtain a set of connectivity matrices (i.e., dFCs), summarizing the temporal evolution of wholebrain FC (Fig. 1A). In this work, we started by exploring six different window lengths $w$, specifically of 7.2, 36, 72, 144, 288, and $576 \mathrm{~s}$ each, and the sets of dFCs associated with them (the number of brain regions is equal to 419). The choice of the shortest window 
length did not consider the recommendation of previous work (50) based on stationarity assumptions; instead, we opted to allow transient nonstationary events at the level of edgewise FC (41) to be fully present in the dFCs at the risk of potential aliasing. The window step $T$ was fixed to $7.2 \mathrm{~s}$ in this study [note that because the repetition time (TR) of HCP is $0.720 \mathrm{~s}, 7.2 \mathrm{~s}$ corresponds to $10 \mathrm{fMRI}$ data points]. Specifically, we studied the evolution of brain fingerprinting across different temporal windows, as detailed in the next section.

\section{Dynamic identification}

The idea on dynamic identification was inspired by recent work on maximization of connectivity fingerprints in human functional connectomes (11). In that work, the authors defined a mathematical object known as "identifiability matrix," which is a similarity matrix encoding the information about the self-similarity (Iself; main diagonal elements) of each subject with herself/himself, across the test/ retest sessions, and the similarity of each subject with the others (or Iothers; off-diagonal elements). The similarity between two functional connectomes was quantified with the Pearson's correlation coefficient between the entries of the connectivity matrices. The difference between Iself and Iothers (denominated "differential identifiability" or "differential identification," Idiff) provides a robust score of the fingerprinting level of a specific dataset (11). This idea needs to be extended in the case of dFC evaluation, because in addition to the test/retest set, the set of dynamic frames of connectivity is estimated (fig. S1, A and B). For a fixed window length $w$, the resulting dynamic identifiability matrix (fig. S1C) $d \mathrm{I}$ is then a block diagonal matrix, where each block represents the self-similarity within the $\mathrm{dFC}$ frames of a specific subject. The off-diagonal blocks, in this representation, encode instead the between-dFC frames similarity across different subjects (dynamic Iothers). Let $S_{M T}=\left\{\mathrm{dFC}_{1}, \mathrm{dFC}_{2}\right.$, $\ldots, \mathrm{dFC}_{N}$ be the set of $\mathrm{dFC}$ frames in the test session for a specific subject $M$. Similarly, let $S_{M R T}$ represent the set of dFC frames in the retest session for the same subject $M$. We can then define the dynamic Iself ( $d$ Iself) for subject $M$ as

$d \operatorname{Iself}(M)=\frac{\sum_{i \in S_{M_{\mathrm{T}}}} \sum_{j \in S_{M_{\mathrm{RT}}}} \operatorname{corr}\left(\mathrm{dFC}_{i}, \mathrm{dFC}_{j}\right)}{\left|S_{M_{\mathrm{T}}}\right| \times\left|S_{M_{\mathrm{RT}}}\right|}=\frac{\sum_{i \in S_{M_{\mathrm{T}}}} \sum_{j \in S_{M_{\mathrm{RT}}}} d I_{i j}}{\left|S_{M_{\mathrm{T}}}\right| \times\left|S_{M_{\mathrm{RT}}}\right|}$

where $\left|S_{M T}\right|,\left|S_{M R T}\right|$ define the cardinalities of the sets. Similarly, let $\left|S_{F T}\right|,\left|S_{F R T}\right|$ define the sets for a different subject $F$. We can define dynamic Iothers as

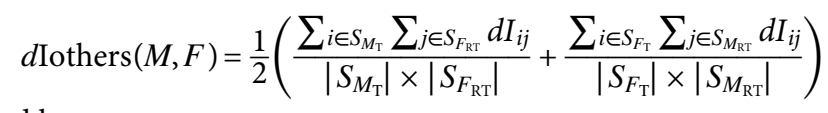

and hence

$$
d \operatorname{Iothers}(M)=\frac{\sum_{F=1}^{S} d \operatorname{Iothers}(M, F)}{S-1}, F \neq M
$$

where the summation is over the total number of subjects $S$ other than $M$. Last, $d$ Idiff for a subject $M$ results in

$$
d \operatorname{Idiff}(M)=d \operatorname{Iself}(M)-d \operatorname{Iothers}(M)
$$

and the average $d$ Idiff

$$
d \text { Idiff }=\frac{\sum_{M=1}^{S} d \operatorname{Idiff}(M)}{S}
$$

Furthermore, we also used the identification scoring method known as SR (6), which can be easily computed in the dynamic domain from $d$ Iself and $d$ Iothers, as the percentage of times $d$ Iself is greater than $d$ Iothers, across subjects (averaged across test-retest and retest-test). We used $d$ Idiff and SR to explore connectivity fingerprints across different window lengths. Note that we first evaluated dynamic identification of the LR and RL connectome pairs separately and then averaged the corresponding LR/RL dynamic identifiability matrices into one.

\section{Null model for identifiability scores}

To assess the statistical significance of the observed differential identifiability and SR scores, we designed the following permutation testing framework: At each iteration of the permutation testing, subjects' test-retest dynamic connectome frames were randomly shuffled, and then differential identifiability and SR were computed on the randomized dynamic identifiability matrix. This procedure was repeated 150 times to generate a "null" distribution for differential identifiability and corresponding SR scores. The observed differential identifiability and SR values were then compared against their corresponding null distribution to determine their significance. Last, the obtained $P$ values were Bonferroni-corrected (51) for multiple comparisons across the six time scales evaluated.

\section{Maximum dynamic dFC frame selection}

The dynamic identification framework described above provides the average behavior of fingerprinting within and between $\mathrm{dFC}$ frames. However, there might be dynamic FC frames where identification is higher than others, which might not be captured by the average behavior depicted by $d$ Idiff. To cover the necessity of that, for each subject, we sorted the dFC frames in test-retest according to their similarity, from highest to lowest, based on their $d$ Iself $_{i j}$ value (see $d$ Iself equation above). We then explored $d$ Iself, $d$ Iothers, and $d$ Idiff when iteratively adding $\mathrm{dFC}$ frames one at the time, starting from the best matching ones and then proceeding based on their similarity values.

\section{Transient (total activation) analysis}

The total activation (TA) framework (19) incorporates two main features for fMRI data processing: (i) Each voxel's BOLD time course is deconvolved from the temporal blur introduced by the hemodynamic response, leading to the activity-inducing signal that is supposed to show block-type activation patterns (without any prior knowledge on the timing and duration of these blocks), and (ii) BOLD signals should show a spatial smoothness, which is supposed to be stronger within anatomical atlas regions than across. With that aim, TA solves a convex optimization problem that consists of a least-squares data-fitting term combined with spatial and temporal regularization terms. TA produces denoised and well-behaving reconstructions of the activity-related, activity-inducing signals, decoupled from the hemodynamics. We used this framework to study dynamic identification properties of $\mathrm{dFC}$ obtained from transient brain activity and compare it to the results obtained with the original dFC frames.

\section{Dynamic edgewise identification}

We quantified the edgewise reliability of individual dynamic FC frames across different temporal windows by evaluating the ICC coefficient (52), similarly to previous work (11). ICC is a widely 
used statistical measure to assess the percent of agreement between units of different groups. It describes how strongly units in the same group resemble each other. The stronger the agreement, the higher its ICC value. We used ICC to quantify to which extent the connectivity value of an edge in an FC frame (i.e., FC value between two brain regions) was consistent across test/retest acquisitions and could separate within- and between-subject data. Specifically, we used the $\operatorname{ICC}(1,1)$ variant, which is usually used to estimate agreement in exact values when sources of error are unspecified $(53,54)$. In other words, the higher the ICC, the higher the "fingerprint" of the edge connectivity. Note that we thresholded the resulting ICC matrices at 0.4 , which is usually a lower limit to define a "good" ICC score $(55,56)$. Last, the nodal strength of the ICC edgewise matrix (i.e., sum over columns, evaluated with and without thresholding the ICC matrix; see also Fig. 4) was used as a "nodal fingerprinting score" of how central each brain region is to connectome identification.

\section{Significance of dynamic edgewise identification}

To better characterize the ICC results in dFCs presented in Fig. 4, we performed a permutation testing analysis. Concretely, we evaluated ICC scores in 100 surrogate datasets where subject labels have been randomly shuffled (fig. S3). Comparing these ICC scores to the original ICC scores presented in Fig. 4A allowed us to evaluate the extent to which the best matching connectivity patterns in test and retest datasets at different time scales are unique to the subjects.

\section{Brain fingerprints and behavior}

A Neurosynth meta-analysis (https://neurosynth.org/) similar to the one implemented in previous studies $(20,21)$ was conducted to assess topic terms associated with brain fingerprints across time scales. First, we created 50 regions of interest binary masks of brain fingerprints at different time scales (from 50-s windows to $770 \mathrm{~s}$, in steps of $15 \mathrm{~s}$ ). The binary masks were obtained by selecting the top 25 percentile of ICC nodal strength at each time step. Each obtained binary mask provides a nodal representation of the brain region "hubs" involved in dynamic fingerprints at a specific time scale. Next, these maps were used as input for the meta-analysis to find significant associations between the ICC hub masks and the brain binary maps related to 50 topic terms common in the neuroimaging literature $(15,16)$. Neurosynth outputs, for each dynamic fingerprint binary mask and topic term binary mask, a nodal $z$ statistic that assesses the similarity between the two maps. Last, we ordered the terms according to the weighted mean of the resulting $z$ statistics for visualization, considering significant any association between dynamic fingerprints and cognitive maps above $z>3.1(20,21)$.

\section{Effects of motion on most identifiable dynamic FC frames}

To test whether top identifiable frames, especially at faster temporal scales, where affected or driven by motion, we performed the following analyses: (i) We evaluated the percentage of censored volumes by tagging fMRI volumes with high framewise displacement scores [here defined as relative root mean square (RMS) distance between frames greater than $>0.2(57,58)$; HCP file: "Movement_RelativeRMS. txt"] across the top identifiable $k$ FC frames. In other words, we aimed to assess how many poor-quality volumes were contributing to the identifiability metrics reported. (ii) We evaluated the edgewise SD and edgewise median for the most identifiable frames across temporal scales, before and after motion scrubbing of the censored volumes.

\section{SUPPLEMENTARY MATERIALS}

Supplementary material for this article is available at https://science.org/doi/10.1126/ sciadv.abj0751

View/request a protocol for this paper from Bio-protocol.

\section{REFERENCES AND NOTES}

1. A. K. Datta, Advances in Fingerprint Technology (CRC Press, 2001).

2. K. Delac, M. Grgic, A survey of biometric recognition methods, in Proceedings of the 46th International Symposium on Electronics in Marine (Elmar-2004) (IEEE, 2004), pp. 184-193.

3. A. Fornito, A. Zalesky, E. Bullmore, Fundamentals of Brain Network Analysis (Academic Press, 2016).

4. D. S. Bassett, O. Sporns, Network neuroscience. Nat. Neurosci. 20, 353-364 (2017).

5. M. D. Fox, M. E. Raichle, Spontaneous fluctuations in brain activity observed with functional magnetic resonance imaging. Nat. Rev. Neurosci. 8, 700-711 (2007).

6. E. S. Finn, X. Shen, D. Scheinost, M. D. Rosenberg, J. Huang, M. M. Chun, X. Papademetris, R. T. Constable, Functional connectome fingerprinting: Identifying individuals using patterns of brain connectivity. Nat. Neurosci. 18, 1664-1671 (2015).

7. O. Miranda-Dominguez, B. D. Mills, S. D. Carpenter, K. A. Grant, C. D. Kroenke, J. T. Nigg, D. A. Fair, Connectotyping: Model based fingerprinting of the functional connectome. PLOS ONE 9, e111048 (2014).

8. M. W. Cole, D. S. Bassett, J. D. Power, T. S. Braver, S. E. Petersen, Intrinsic and task-evoked network architectures of the human brain. Neuron 83, 238-251 (2014).

9. K. Abbas, E. Amico, D. O. Svaldi, U. Tipnis, D. A. Duong-Tran, M. Liu, M. Rajapandian, J. Harezlak, B. M. Ances, J. Goñi, GEFF: Graph embedding for functional fingerprinting Neuroimage 221, 117181 (2020).

10. A. Fornito, A. Zalesky, M. Breakspear, The connectomics of brain disorders. Nat. Rev. Neurosci. 16, 159-172 (2015).

11. E. Amico, J. Goñi, The quest for identifiability in human functional connectomes. Sci. Rep. 8, 8254 (2018).

12. S. Sadaghiani, J. Wirsich, Intrinsic connectome organization across temporal scales: New insights from cross-modal approaches. Netw. Neurosci. 4, 1-29 (2020).

13. G. Deco, V. K. Jirsa, A. R. McIntosh, Emerging concepts for the dynamical organization of resting-state activity in the brain. Nat. Rev. Neurosci. 12, 43-56 (2011).

14. U. Hasson, E. Yang, I. Vallines, D. J. Heeger, N. Rubin, A hierarchy of temporal receptive windows in human cortex. J. Neurosci. 28, 2539-2550 (2008).

15. R. M. Birn, E. K. Molloy, R. Patriat, T. Parker, T. B. Meier, G. R. Kirk, V. A. Nair, M. E. Meyerand, V. Prabhakaran, The effect of scan length on the reliability of resting-state fMRI connectivity estimates. Neuroimage 83, 550-558 (2013).

16. C. Horien, X. Shen, D. Scheinost, R. T. Constable, The individual functional connectome is unique and stable over months to years. Neuroimage 189, 676-687 (2019).

17. J. Liu, X. Liao, M. Xia, Y. He, Chronnectome fingerprinting: Identifying individuals and predicting higher cognitive functions using dynamic brain connectivity patterns. Hum. Brain Mapp. 39, 902-915 (2018).

18. S. S. Menon, K. Krishnamurthy, A comparison of static and dynamic functional connectivities for identifying subjects and biological sex using intrinsic individual brain connectivity. Sci. Rep. 9, 5729 (2019).

19. F. I. Karahanoğlu, C. Caballero-Gaudes, F. Lazeyras, D. Van de Ville, Total activation: fMRI deconvolution through spatio-temporal regularization. Neuroimage 73, 121-134 (2013).

20. M. G. Preti, D. Van De Ville, Decoupling of brain function from structure reveals regional behavioral specialization in humans. Nat. Commun. 10, 4747 (2019).

21. D. S. Margulies, S. S. Ghosh, A. Goulas, M. Falkiewicz, J. M. Huntenburg, G. Langs, G. Bezgin, S. B. Eickhoff, F. X. Castellanos, M. Petrides, E. Jefferies, J. Smallwood, Situating the default-mode network along a principal gradient of macroscale cortical organization. Proc. Natl. Acad. Sci. U.S.A. 113, 12574-12579 (2016).

22. D. C. Van Essen, S. M. Smith, D. M. Barch, T. E. J. Behrens, E. Yacoub, K. Ugurbil; WU-Minn HCP Consortium, The WU-Minn Human Connectome Project: An overview. Neuroimage 80, 62-79 (2013)

23. T. E. Nichols, S. Das, S. B. Eickhoff, A. C. Evans, T. Glatard, M. Hanke, N. Kriegeskorte, M. P. Milham, R. A. Poldrack, J.-B. Poline, E. Proal, B. Thirion, D. C. Van Essen, T. White, B. T. T. Yeo, Best practices in data analysis and sharing in neuroimaging using MRI. Nat. Neurosci. 20, 299-303 (2017).

24. E. Amico, D. Marinazzo, C. Di Perri, L. Heine, J. Annen, C. Martial, M. Dzemidzic, M. Kirsch, V. Bonhomme, S. Laureys, J. Goñi, Mapping the functional connectome traits of levels of consciousness. Neuroimage 148, 201-211 (2017).

25. P. M. Thompson, T. Ge, D. C. Glahn, N. Jahanshad, T. E. Nichols, Genetics of the connectome. Neuroimage 80, 475-488 (2013).

26. F. Z. Esfahlani, Y. Jo, J. Faskowitz, L. Byrge, D. P. Kennedy, O. Sporns, R. F. Betzel, High-amplitude cofluctuations in cortical activity drive functional connectivity. Proc. Natl. Acad. Sci. U.S.A. 117, 28393-28401 (2020). 
27. O. Sporns, J. Faskowitz, A. S. Teixeira, S. A. Cutts, R. F. Betzel, Dynamic expression of brain functional systems disclosed by fine-scale analysis of edge time series. Netw. Neurosci. $\mathbf{5}$ 405-433 (2021).

28. G. Buzsáki, A. Draguhn, Neuronal oscillations in cortical networks. Science $\mathbf{3 0 4}$ 1926-1929 (2004).

29. N. K. Logothetis, O. Eschenko, Y. Murayama, M. Augath, T. Steudel, H. C. Evrard M. Besserve, A. Oeltermann, Hippocampal-cortical interaction during periods of subcortical silence. Nature 491, 547-553 (2012).

30. J. Gonzalez-Castillo, D. A. Handwerker, M. E. Robinson, C. W. Hoy, L. C. Buchanan Z. S. Saad, P. A. Bandettini, The spatial structure of resting state connectivity stability on the scale of minutes. Front. Neurosci. 8, 138 (2014).

31. J. L. Robinson, A. R. Laird, D. C. Glahn, W. R. Lovallo, P. T. Fox, Metaanalytic connectivity modeling: Delineating the functional connectivity of the human amygdala. Hum. Brain Mapp. 31, 173-184 (2010).

32. W. W. Seeley, V. Menon, A. F. Schatzberg, J. Keller, G. H. Glover, H. Kenna, A. L. Reiss, M. D. Greicius, Dissociable intrinsic connectivity networks for salience processing and executive control. J. Neurosci. 27, 2349-2356 (2007).

33. R. Gao, R. L. van den Brink, T. Pfeffer, B. Voytek, Neuronal timescales are functionally dynamic and shaped by cortical microarchitecture. eLife 9, e61277 (2020).

34. S. J. Kiebel, J. Daunizeau, K. J. Friston, A hierarchy of time-scales and the brain. PLOS Comput. Biol. 4, e1000209 (2008).

35. C. Baldassano, J. Chen, A. Zadbood, J. W. Pillow, U. Hasson, K. A. Norman, Discovering event structure in continuous narrative perception and memory. Neuron 95, 709-721.e5 (2017).

36. R. Chaudhuri, K. Knoblauch, M.-A. Gariel, H. Kennedy, X.-J. Wang, A large-scale circuit mechanism for hierarchical dynamical processing in the primate cortex. Neuron $\mathbf{8 8}$, 419-431 (2015).

37. T. Yarkoni, R. A. Poldrack, T. E. Nichols, D. C. Van Essen, T. D. Wager, Large-scale automated synthesis of human functional neuroimaging data. Nat. Methods 8, 665-670 (2011)

38. M. Golesorkhi, J. Gomez-Pilar, S. Tumati, M. Fraser, G. Northoff, Temporal hierarchy of intrinsic neural timescales converges with spatial core-periphery organization. Commun. Biol. 4, 277 (2021).

39. A. Kucyi, A. Tambini, S. Sadaghiani, S. Keilholz, J. R. Cohen, Spontaneous cognitive processes and the behavioral validation of time-varying brain connectivity. Netw. Neurosci. 2, 397-417 (2018).

40. C. Cioli, H. Abdi, D. Beaton, Y. Burnod, S. Mesmoudi, Differences in human cortical gene expression match the temporal properties of large-scale functional networks. PLOS ONE 9, e115913 (2014).

41. J. Faskowitz, F. Z. Esfahlani, Y. Jo, O. Sporns, R. F. Betzel, Edge-centric functional network representations of human cerebral cortex reveal overlapping system-level architecture. Nat. Neurosci. 23, 1644-1654 (2020).

42. M. G. Preti, T. A. Bolton, D. Van De Ville, The dynamic functional connectome: State-of-the-art and perspectives. Neuroimage 160, 41-54 (2017)

43. T. A. W. Bolton, V. Kebets, E. Glerean, D. Zöller, J. Li, B. T. T. Yeo, C. Caballero-Gaudes, D. Van De Ville, Agito ergo sum: Correlates of spatio-temporal motion characteristics during fMRI. Neuroimage 209, 116433 (2020).

44. J. D. Power, A. Mitra, T. O. Laumann, A. Z. Snyder, B. L. Schlaggar, S. E. Petersen, Methods to detect, characterize, and remove motion artifact in resting state fMRI. Neuroimage $\mathbf{8 4}$ 320-341 (2014).

45. R. Liégeois, A. Santos, V. Matta, D. Van De Ville, A. H. Sayed, Revisiting correlation-based functional connectivity and its relationship with structural connectivity. Netw. Neurosci. 4 1235-1251 (2020).

46. D. C. Van Essen, K. Ugurbil, E. Auerbach, D. Barch, T. E. J. Behrens, R. Bucholz, A. Chang, L. Chen, M. Corbetta, S. W. Curtiss, S. Della Penna, D. Feinberg, M. F. Glasser, N. Harel, A. C. Heath, L. Larson-Prior, D. Marcus, G. Michalareas, S. Moeller, R. Oostenveld, S. E. Petersen, F. Prior, B. L. Schlaggar, S. M. Smith, A. Z. Snyder, J. Xu, E. Yacoub; WU-Minn HCP Consortium, The Human Connectome Project: A data acquisition perspective. Neuroimage 62, 2222-2231 (2012).

47. M. F. Glasser, S. N. Sotiropoulos, J. A. Wilson, T. S. Coalson, B. Fischl, J. L. Andersson, J. Xu, S. Jbabdi, M. Webster, J. R. Polimeni, D. C. Van Essen, M. Jenkinson; WU-Minn HCP Consortium, The minimal preprocessing pipelines for the Human Connectome Project. Neuroimage 80, 105-124 (2013).
48. S. M. Smith, C. F. Beckmann, J. Andersson, E. J. Auerbach, J. Bijsterbosch, G. Douaud, E. Duff, D. A. Feinberg, L. Griffanti, M. P. Harms, M. Kelly, T. Laumann, K. L. Miller, S. Moeller, S. Petersen, J. Power, G. Salimi-Khorshidi, A. Z. Snyder, A. T. Vu, M. W. Woolrich J. Xu, E. Yacoub, K. Uğurbil, D. C. Van Essen, M. F. Glasser; WU-Minn HCP Consortium, Resting-state fMRI in the Human Connectome Project. Neuroimage 80, 144-168 (2013).

49. A. Schaefer, R. Kong, E. M. Gordon, T. O. Laumann, X.-N. Zuo, A. J. Holmes, S. B. Eickhoff, B. T. T. Yeo, Local-global parcellation of the human cerebral cortex from intrinsic functional connectivity MRI. Cereb. Cortex 28, 3095-3114 (2018).

50. N. Leonardi, D. Van De Ville, On spurious and real fluctuations of dynamic functional connectivity during rest. Neuroimage 104, 430-436 (2015).

51. T. E. Nichols, A. P. Holmes, Nonparametric permutation tests for functional neuroimaging: A primer with examples. Hum. Brain Mapp. 15, 1-25 (2002).

52. J. J. Bartko, The intraclass correlation coefficient as a measure of reliability. Psychol. Rep. 19, 3-11 (1966).

53. S. Noble, D. Scheinost, R. T. Constable, A decade of test-retest reliability of functional connectivity: A systematic review and meta-analysis. Neuroimage 203, 116157 (2019)

54. S. Bari, E. Amico, N. Vike, T. M. Talavage, J. Goñi, Uncovering multi-site identifiability based on resting-state functional connectomes. Neuroimage 202, 115967 (2019).

55. P. E. Shrout, J. L. Fleiss, Intraclass correlations: Uses in assessing rater reliability. Psychol. Bull. 86, 420-428 (1979).

56. K. O. McGraw, S. P. Wong, Forming inferences about some intraclass correlation coefficients. Psychol. Methods 1, 30-46 (1996).

57. M. Jenkinson, P. Bannister, M. Brady, S. Smith, Improved optimization for the robust and accurate linear registration and motion correction of brain images. Neuroimage 17, 825-841 (2002).

58. J. Bijsterbosch, S. Harrison, E. Duff, F. Alfaro-Almagro, M. Woolrich, S. Smith, Investigations into within- and between-subject resting-state amplitude variations. Neuroimage 159, 57-69 (2017).

59. B. T. T. Yeo, F. M. Krienen, J. Sepulcre, M. R. Sabuncu, D. Lashkari, M. Hollinshead, J. L. Roffman, J. W. Smoller, L. Zöllei, J. R. Polimeni, B. Fischl, H. Liu, R. L. Buckner, The organization of the human cerebral cortex estimated by intrinsic functional connectivity. J. Neurophysiol. 106, 1125-1165 (2011).

Acknowledgments: We thank E. De Falco for insightful discussions. Funding: Data were provided (in part) by the Human Connectome Project, WU-Minn Consortium (principal investigators: D. V. Essen and K. Ugurbil; 1U54MH091657) funded by the $16 \mathrm{NIH}$ Institutes and Centers that support the NIH Blueprint for Neuroscience Research and by the McDonnell Center for Systems Neuroscience at Washington University. E.A. acknowledges financial support from the SNSF Ambizione project "Fingerprinting the brain: Network science to extract features of cognition, behavior and dysfunction" (grant number PZ00P2 185716). M.G.P. was supported by the CIBM Center for Biomedical Imaging, a Swiss research center of excellence founded and supported by Lausanne University Hospital (CHUV), University of Lausanne (UNIL), Ecole polytechnique fédérale de Lausanne (EPFL), University of Geneva (UNIGE), and Geneva University Hospitals (HUG). R.L. was supported by the National Centre of Competence in Research-Evolving Language (grant number 51NF40_180888). Author contributions: E.A., M.G.P., and R.L. processed the data. D.V.D.V. and E.A. conceptualized the study. E.A. designed the framework and performed the connectivity analyses. E.A. and Y.F. performed the activity-inducing analysis. All authors interpreted the results and wrote the manuscript. Competing interests: The authors declare that they have no competing interests. Data and materials availability: All data needed to evaluate the conclusions in the paper are present in the paper and/or the Supplementary Materials. The code (in MATLAB) and sample connectomes used for this analysis are available on Zenodo (10.5281/zenodo.5376087) as well as a maintained version on E.A.'s GitHub page (https://github.com/eamico)

Submitted 19 April 2021

Accepted 20 August 2021

Published 15 October 2021

10.1126/sciadv.abj0751

Citation: D. Van De Ville, Y. Farouj, M. G. Preti, R. Liégeois, E. Amico, When makes you unique: Temporality of the human brain fingerprint. Sci. Adv. 7, eabj0751 (2021). 